\title{
On the Statistical Decorrelation of the Wavelet Packet Coefficients of a Band-Limited Wide-Sense Stationary Random Process
}

\author{
Abdourrahmane M. Atto* Dominique Pastor ${ }^{\dagger}$
}

Alexandru Isar $\ddagger$

\begin{abstract}
This paper is a contribution to the analysis of the statistical correlation of the wavelet packet coefficients resulting from the decomposition of a random process, stationary in the wide-sense, whose power spectral density is bounded with support in $[-\pi, \pi]$.

Consider two quadrature mirror filters $(\mathrm{QMF})$ that depend on a parameter $r$, such that these filters tend almost everywhere to the Shannon QMF when $r$ increases. The parameter $r$ is called the order of the QMF under consideration. The order of the Daubechies filters (resp. the Battle-Lemarié filters) is the number of vanishing moments of the wavelet function (resp. the spline order of the scaling function).

Given any decomposition path in the wavelet packet tree, the wavelet packet coefficients are proved to decorrelate for every packet associated with a large enough resolution level, provided that the QMF order is large enough and above a value that depends on this wavelet packet.

Another consequence of our derivation is that, when the coefficients associated with a given wavelet packet are approximately decorrelated, the value of the autocorrelation function of these coefficients at lag 0 is close to the value taken by the power spectral density of the decomposed process at a specific point. This specific point depends on the path followed in the wavelet packet tree to attain the wavelet packet under consideration.

Some simulations highlight the good quality of the "whitening" effect that can be obtained in practical cases.
\end{abstract}

Keywords Wavelet packets, wide-sense stationary random process, autocorrelation function, Daubechies filters, Battle-Lemarié filters, mean-square integral, spectral representation of a process.

\section{Introduction}

The transforms that map an original continuous-time random process into a set of uncorrelated random variables can be regarded as optimal discretisations in

*ENST Bretagne, am.atto@enst-bretagne.fr

†ENST Bretagne, dominique.pastor@enst-bretagne.fr

‡UPT Romania, alexandru.isar@etc.upt.ro 
the sense that the analysis of a random sequence whose elements are not correlated is much simpler than the analysis of a continuous-time random process. Therefore, such transforms are of great practical interest in signal processing and communications applications. The Karhunen-Loève (KL) expansion is a typical example of such an optimal discretisation. It applies to any Wide-SenseStationary (WSS) random process, that is, any Hilbertian or second-order process $X(t)\left(\mathbb{E}\left[|X(t)|^{2}\right]<\infty\right)$ whose correlation function $R(t, s)=\mathbb{E}[X(t) \overline{X(s)}]$ depends only on the time-increment $t-s$ between its two arguments.

The analysis of time-series through the Wavelet transform has thus received much interest in recent years. Many authors have studied various aspects of the statistical correlation of the wavelet coefficients (see $[1,2,3,4,5,6,7]$, amongst others). These works highlight that, for many stationary and non-stationary input random processes, the between-scale and within-scale coefficients returned by a Discrete Wavelet Transform (DWT) tend to decorrelate as the resolution level increases; for further details, the reader may refer to the very nice overview given in [7]. The DWT can thus be regarded as a "nearly" optimal discretisation. For WSS processes, it is a relevant alternative to KL theory because, unlike the KL expansion, the DWT expansion achieves the "whitening effect" without solving any eigen equation and without having to assume that the process is time-limited, a constraint of importance in KL theory. For instance, in [8], the DWT whitening effect is used to design a telecommunication receiver robust in presence of WSS noise; this system performs without knowing the noise Power Spectral Density (PSD) and it yields binary error rates comparable to those achieved by the optimal receiver based on the noise KL expansion. Note also that when the autocorrelation function of a given WSS process is known, it is even possible to construct a non-orthogonal wavelet basis in terms of which the process can be expanded with uncorrelated coefficients; the construction of this basis still requires no eigen equation to solve and does not need us to assume that the process is time-limited [9].

In addition to the results recalled above and dedicated to the DWT, the present paper addresses the Discrete Wavelet Packet Transform (DWPT) of a zero-mean WSS random process. In particular, it proposes an analysis of the whitening effect that the DWPT can achieve in any path when the resolution level increases. This analysis is motivated by the following facts.

Let $\left(c_{j, n}[k]\right)_{k \in \mathbb{Z}}$ be the sequence of the DWPT within-scale coefficients that are returned at node $(j, n)$ of the wavelet packet tree by decomposing the input WSS random process; $j$ is the resolution level and $n$ is the shift parameter valued in $\left\{0,1, \ldots, 2^{j}-1\right\}$ (see section 2.2 ).

If $n$ is constant with $j$ or if the value of $n$ depends on $j$ but remains upperbounded by a constant independent of $j$, it follows from [10] that, when $j$ tends to infinity, the coefficients $\left(c_{j, n}[k]\right)_{k \in \mathbb{Z}}$ tend to decorrelate and that the autocorrelation function at lag 0 of the discrete random process $\left(c_{j, n}[k]\right)_{k \in \mathbb{Z}}$ tends to the value of the PSD of the input random process at the origin.

However, as explained below, when $n$ is not a fixed constant or does not remain upper-bounded by a value independent of $j$, the analysis of the autocorrelation function of $\left(c_{j, n}[k]\right)_{k \in \mathbb{Z}}$ becomes significantly more intricate: in this case, given an arbitrary pair of quadrature mirror filters (QMF), we cannot guarantee that increasing only the resolution level is sufficient to obtain asymptotically decorrelated wavelet packet coefficients. Moreover, even when the whitening effect is guaranteed, the value of the asymptotic autocorrelation 
function at lag 0 of the discrete random process $\left(c_{j, n}[k]\right)_{k \in \mathbb{Z}}$ is no longer the value of the PSD at the origin but actually depends on the sequence of the values of $n$ in the DWPT path followed when $j$ varies; for example, if we choose $n=2^{j}-1$ at every resolution level $j$, the value of the asymptotic autocorrelation function at the origin is the value taken by the PSD at $\pi$.

The principal contribution of this paper is thus theorem 1 . The asymptotic decorrelation of the wavelet packet coefficients stated by this theorem for a large class of WSS random processes encountered in practice is obtained by considering QMF $h_{0}^{[r]}$ and $h_{1}^{[r]}$ whose Fourier transforms $H_{0}^{[r]}$ and $H_{1}^{[r]}$ tend almost everywhere to the Fourier transforms of the Shannon QMF when $r$ increases. The parameter $r$ is hereafter called the order of the QMF $h_{0}^{[r]}$ and $h_{1}^{[r]}$. If $h_{0}^{[r]}$ and $h_{1}^{[r]}$ are Daubechies QMF, the order $r$ is the number of vanishing moments of the wavelet function associated with $H_{0}^{[r]}$; if these QMF are Battle-Lemarié filters, $r$ is the spline order of the scaling function associated with $H_{0}^{[r]}$.

Several papers have already stressed the importance of parameters such as the order $r$ for analysing the statistical correlation of the DWT and DWPT coefficients. For a fractional Brownian motion, [1] shows that the larger the number of wavelet vanishing moments, the more decorrelated the wavelet coefficients. In [11], an asymptotic within-scale nearly-whiteness inequality is achieved for wavelet packet coefficients when the regularity of the scaling function increases. Recently, [7] has highlighted the role played by the length $L$ of the impulse response of the Daubechies filters to obtain decorrelated between-scale wavelet coefficients: the covariance of the between-scale coefficients of some stationary process tends to 0 at rate $L^{-1 / 4}$ as $L$ tends to infinity.

This paper is organized as follows. In section 2 , we recall basic results concerning the wavelet packet decomposition of a random process. In particular, we give the expressions of the autocorrelation functions of the discrete sequences formed by the wavelet packet coefficients. The convergence of these function sequences when the resolution level tends to infinity is studied in section 3, and is achieved in two steps. In section 3.2 , the asymptotic decorrelation is established for the Shannon wavelet packet decomposition, which employs the Shannon QMF that are ideal filters. In section 3.3, we consider QMF for which the above notion of order makes sense. We use the convergence of such filters to the Shannon filters when the order increases to prove that the wavelet packet coefficients tend to be decorrelated when both the resolution level and the order of the QMF are large enough; the order must be chosen according to the resolution level. In section 4 , we present some experimental results to illustrate the role played by the resolution level and the order of the QMF in the decorrelation process. Finally, we conclude in section 5 .

\section{Discrete wavelet packet decomposition of a wide-sense stationary random process}

In section 2.1, we present some aspects concerning wavelet packet analysis. We adopt the same notations as [12]. Section 2.2 gives the decomposition of a WSS random process and the expressions of the autocorrelation functions of its wavelet packet coefficients. 


\subsection{Discrete wavelet packet analysis}

Let $\Phi$ be a function such that $\left\{\tau_{k} \Phi: k \in \mathbb{Z}\right\}$ is an orthonormal system of $L^{2}(\mathbb{R})$, where $\tau_{k} \Phi: t \longmapsto \Phi(t-k)$. Let $\mathbf{U}$ be the closure of the space spanned by this orthonormal system.

Consider two QMF $h_{0}$ and $h_{1}$ and define

$$
\left\{\begin{array}{l}
H_{0}(\omega)=\frac{1}{\sqrt{2}} \sum_{\ell \in \mathbb{Z}} h_{0}[\ell] e^{-i \ell \omega}, \\
H_{1}(\omega)=\frac{1}{\sqrt{2}} \sum_{\ell \in \mathbb{Z}} h_{1}[\ell] e^{-i \ell \omega} .
\end{array}\right.
$$

The functions $H_{0}$ and $H_{1}$ are, up to the factor $1 / \sqrt{2}$, the Fourier transforms of $h_{0}$ and $h_{1}$, respectively. The quadrature mirror condition is equivalent to the unitarity of the matrix

$$
M(\omega)=\left(\begin{array}{cc}
H_{0}(\omega) & H_{1}(\omega) \\
H_{0}(\omega+\pi) & H_{1}(\omega+\pi)
\end{array}\right)
$$

for every $\omega$. We assume that the functions $H_{0}$ and $H_{1}$ are such that

$$
H_{1}(\omega)=e^{-i \omega} \overline{H_{0}(\omega+\pi)} .
$$

where $\bar{z}$ stands for the complex conjugate of $z$.

We define the sequence $\left(W_{n}\right)_{n \geq 0}$ of elements of $L^{2}(\mathbb{R})$ by :

$$
\left\{\begin{array}{l}
W_{0}(t)=\sqrt{2} \sum_{\ell \in \mathbb{Z}} h_{0}[\ell] \Phi(2 t-\ell) \\
W_{1}(t)=\sqrt{2} \sum_{\ell \in \mathbb{Z}} h_{1}[\ell] \Phi(2 t-\ell),
\end{array}\right.
$$

and by setting, for all $n \geq 1$,

$$
\left\{\begin{array}{l}
W_{2 n}(t)=\sqrt{2} \sum_{\ell \in \mathbb{Z}} h_{0}[\ell] W_{n}(2 t-\ell) \\
W_{2 n+1}(t)=\sqrt{2} \sum_{\ell \in \mathbb{Z}} h_{1}[\ell] W_{n}(2 t-\ell) .
\end{array}\right.
$$

The wavelet packet functions are then defined by

$$
W_{j, n}(t)=2^{-j / 2} W_{n}\left(2^{-j} t\right)
$$

For $j \geq 1$ and $k \in \mathbb{Z}$, we put $W_{j, n, k}=\tau_{2^{j} k} W_{j, n}$, that is,

$$
W_{j, n, k}(t)=2^{-j / 2} W_{n}\left(2^{-j} t-k\right)
$$

The set $\left\{W_{j, n, k}: k \in \mathbb{Z}\right\}$ is orthonormal. With a slight abuse of language, the closure of the space spanned by $\left\{W_{j, n, k}: k \in \mathbb{Z}\right\}$ will hereafter be called the packet $\mathbf{W}_{j, n}$.

The wavelet packet decomposition of the function space $\mathbf{U}$ is obtained by recursively applying the so-called splitting lemma (see [13], for example). We can thus write that

$$
\mathbf{U}=\mathbf{W}_{1,0} \oplus \mathbf{W}_{1,1},
$$

and

$$
\mathbf{W}_{j, n}=\mathbf{W}_{j+1,2 n} \oplus \mathbf{W}_{j+1,2 n+1},
$$


for every $j=1,2, \cdots$, and every $n \in I_{j}$ with

$$
I_{j}=\left\{0,1, \cdots, 2^{j}-1\right\} .
$$

The sets $\left\{W_{j+1,2 n, k}: k \in \mathbb{Z}\right\}$ and $\left\{W_{j+1,2 n+1, k}: k \in \mathbb{Z}\right\}$ are orthonormal bases of the vector spaces $\mathbf{W}_{j+1,2 n}$ and $\mathbf{W}_{j+1,2 n+1}$, respectively. The wavelet packet tree of figure 1 illustrates such a decomposition.

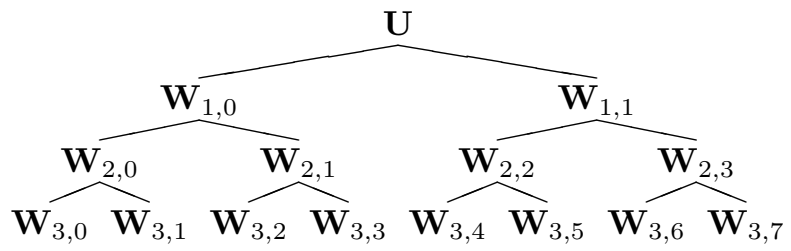

Figure 1: Wavelet packet decomposition tree down to resolution level $j=3$.

The projection of a function $f \in \mathbf{U}$ on $\mathbf{W}_{j, n}$ yields the coefficients

$$
c_{j, n}[k]=\int_{\mathbb{R}} f(t) W_{j, n, k}(t) d t .
$$

Remark 1 With respect to what follows, it is worth emphasizing that the decomposition concerns an arbitrary space $\mathbf{U}$ generated by the translated versions of $\Phi$. Therefore, the function $\Phi$ is not necessarily the scaling function associated with the low-pass filter $H_{0}$. If $\Phi$ is this scaling function, we have $W_{0}=\Phi$ in (4).

In practice, when the input data of the wavelet packet decomposition are the samples of some function that satisfies Shannon's sampling theorem, we implicitly use the wavelet packet decomposition of the space $\mathbf{U}=\mathbf{U}^{S}$ of those elements of $L^{2}(\mathbb{R})$ that have their Fourier transforms with support in $[-\pi, \pi]$. The elements of $\mathbf{U}^{S}$ are said to be band-limited functions. According to Shannon's sampling theorem, $\mathbf{U}^{S}$ is the space spanned by the translated versions of $\Phi^{S}=$ sinc.

\subsection{Discrete wavelet packet decomposition of a second- order WSS random process}

Let $(\Omega, \mathcal{A}, P)$ be a probability space, where $P$ is a probability measure on the elements of $\mathcal{A}$, and let $X: \mathbb{R} \times \Omega \longrightarrow \mathbb{R}$ be a second-order random process:

$$
\|X\|_{L^{2}(\Omega)}^{2}=\mathbb{E}\left[|X|^{2}\right]=\int_{\Omega}|X|^{2} d P<\infty .
$$

We assume that $X$ is zero-mean and continuous in quadratic mean. The autocorrelation function of $X$, denoted by $R$, is a continuous function. This function is defined by

$$
R(t, s)=\langle X(t), X(s)\rangle_{L^{2}(\Omega)}=\mathbb{E}[X(t) \overline{X(s)}] .
$$

The wavelet packet decomposition of $X$ returns, at level $j$ and for $n \in I_{j}$, the random variables

$$
c_{j, n}[k]=\int_{\mathbb{R}} X(t) W_{j, n, k}(t) d t, \quad k \in \mathbb{Z},
$$


provided that the Riemann integral

$$
\iint_{\mathbb{R}^{2}} R(t, s) W_{j, n, k}(t) W_{j, n, k}(s) d t d s
$$

exists (see appendix A). The discrete random process $\left(c_{j, n}[k]\right)_{k \in \mathbb{Z}}$, where $c_{j, n}[k]$ is defined by the mean-square integral (13), represents the sequence of the coefficients of $X$ on the packet $\mathbf{W}_{j, n}$.

Remark 2 According to the splitting lemma (9), $\mathbf{W}_{j, n}$ is decomposed into two packets $\mathbf{W}_{j+1,2 n}$ and $\mathbf{W}_{j+1,2 n+1}$. For $\epsilon \in\{0,1\}$, the coefficients of $X$ on the packet $\mathbf{W}_{j+1,2 n+\epsilon}$ are defined by

$$
c_{j+1,2 n+\epsilon}[k]=\int_{\mathbb{R}} X(t) W_{j+1,2 n+\epsilon, k}(t) d t .
$$

We have (see appendix B),

$$
W_{j+1,2 n+\epsilon, k}=\sum_{\ell \in \mathbb{Z}} h_{\epsilon}[\ell-2 k] W_{j, n, \ell}
$$

and then, we can write, with convergence in $L^{2}(\Omega)$,

$$
c_{j+1,2 n+\epsilon}[k]=\sum_{\ell \in \mathbb{Z}} h_{\epsilon}[\ell-2 k] c_{j, n}[\ell] .
$$

Thus, we can obtain the wavelet packet coefficients of a second-order random process by applying Mallat's algorithm based on convolution and downsampling [14]. The principle of the decomposition is then the same as that used to decompose functions of $L^{2}(\mathbb{R})$, the only difference being the kind of convergence involved.

Now, let $R_{j, n}$ stand for the autocorrelation function of the discrete random process $c_{j, n}$ defined by (13). We have

$$
\begin{aligned}
R_{j, n}[k, \ell] & =\mathbb{E}\left[c_{j, n}[k] \overline{c_{j, n}[\ell]}\right] \\
& =\mathbb{E}\left[\int_{\mathbb{R}} X(t) W_{j, n, k}(t) d t \int_{\mathbb{R}} \overline{X(s) W_{j, n, \ell}(s)} d s\right] \\
& =\iint_{\mathbb{R}^{2}} R(t, s) W_{j, n, k}(t) W_{j, n, \ell}(s) d t d s .
\end{aligned}
$$

Assume that $X$ is a WSS random process. As usual, we write that $R(t, s)=$ $R(t-s)$. We also assume that $X$ has a Power Spectral Density (PSD) $\gamma$, which is the Fourier transform of $R$, and that $\gamma \in L^{\infty}(\mathbb{R})$.

If we denote by $\widehat{f}$ the Fourier transform of any element $f$ of $L^{1}(\mathbb{R})$ or $L^{2}(\mathbb{R})$, it follows from (18) and appendix C, that the discrete random process $c_{j, n}$ is WSS and that

$$
R_{j, n}[m]=\frac{1}{2 \pi} \int_{\mathbb{R}} \gamma\left(\frac{\omega}{2^{j}}\right)\left|\widehat{W_{n}}(\omega)\right|^{2} e^{i m \omega} d \omega .
$$

where, with the same abuse of language as above, $R_{j, n}[k-\ell]=R_{j, n}[k, \ell]$. This autocorrelation function can also be written

$$
R_{j, n}[m]=\frac{1}{2 \pi} \int_{\mathbb{R}} \gamma(\omega)\left|2^{j / 2} \widehat{W_{n}}\left(2^{j} \omega\right)\right|^{2} e^{i 2^{j} m \omega} d \omega
$$


With an easy change of variable, we obtain

$$
R_{j, n}[m]=\frac{1}{2 \pi} \int_{\mathbb{R}} \gamma(\omega)\left|\widehat{W_{j, n}}(\omega)\right|^{2} e^{i 2^{j} m \omega} d \omega,
$$

which will prove useful in the sequel.

\section{Asymptotic decorrelation of the wavelet packet coefficients of a band-limited wide-sense sta- tionary random process}

Our purpose is to analyse the behaviour of the autocorrelation functions (21) when the resolution level $j$ tends to infinity.

If $n$ is constant, Lebesgue's dominated convergence theorem can be used to compute the limit of $R_{j, n}[m]$ when $j$ tends to infinity. More precisely, if we assume that the PSD $\gamma$ of $X$ is an element of $L^{\infty}(\mathbb{R})$, it follows from (19) that

$$
\left|R_{j, n}[m]\right| \leq\|\gamma\|_{\infty}\left\|W_{n}\right\|_{L^{2}(\mathbb{R})}^{2}=\|\gamma\|_{\infty},
$$

and therefore, if $n$ is constant, we have from (19):

$$
\lim _{j \rightarrow+\infty} R_{j, n}[m]=\frac{1}{2 \pi} \gamma(0) \int_{\mathbb{R}}\left|\widehat{W}_{n}(\omega)\right|^{2} e^{i m \omega} d \omega .
$$

But

$$
\int_{\mathbb{R}}\left|\widehat{W}_{n}(\omega)\right|^{2} e^{i m \omega} d \omega=2 \pi\left\langle\tau_{m} W_{n}, W_{n}\right\rangle=2 \pi \delta[m],
$$

where

$$
\delta[m]=\left\{\begin{array}{lll}
1 & \text { if } & m=0 \\
0 & \text { if } & m \neq 0
\end{array}\right.
$$

and then

$$
\lim _{j \rightarrow+\infty} R_{j, n}[m]=\gamma(0) \delta[m] .
$$

The result thus obtained is that given in [10] and is partially proved in [15]. Note that (26) embraces the cases $n=0$ and $n=1$, which correspond to the approximation coefficients and the detail coefficients of the DWT, respectively.

The situation becomes more intricate if $n$ is a function of $j$. For instance, if we choose $n=2^{j}-1$ for all $j>0$ or $n=2^{j-L}$ where $L \in \mathbb{N}$, the behaviour of $R_{j, n}[m]$ when $j$ tends to infinity is no longer a straightforward consequence of Lebesgue's dominated convergence theorem.

The approach proposed below is valid whether $n$ depends on $j$ or not. This approach concerns the case where the random process $X$ is second-order, zeromean, WSS, continuous in quadratic mean, with PSD $\gamma \in L^{\infty}([-\pi, \pi])$. Therefore $X$ is a band-limited random process, and we have (see proof in appendix D),

$$
\int_{\mathbb{R}} X(t) \Phi^{S}(t-k) d t=X[k] .
$$

Consequently, $\mathbf{U}^{S}$ is the natural representation space of such a random process. Note that according to (27), we can initialise the decomposition with the samples 
$(X[k])_{k}$, by setting $c_{0,0}[k]=X[k]$, and calculating the wavelet packet coefficients with the recurrence described by relation (17). From now on, we will assume that the wavelet packet decomposition concerns the space $\mathbf{U}^{S}$.

Before detailing this approach, the next subsection reminds the reader of a useful description of an arbitrary sequence $\left(\mathbf{W}_{j, n}\right)_{j \geq 1}$ of wavelet packets by means of a binary sequence [16]. Then, in subsection 3.2, we treat the case where the quadrature mirror filters are the ideal low and high pass filters of the Shannon decomposition. The general case is addressed in subsection 3.3.

\subsection{The binary sequence associated with a given sequence of wavelet packets}

Let $\kappa=\left(\epsilon_{\ell}\right)_{\ell} \in\{0,1\}^{\mathbb{N}}$ be an infinite binary sequence. For any natural number $j$, let $n_{j}(\kappa)$, in short $n_{j}$, stand for the non negative integer

$$
n_{j}=n_{j}(\kappa)=\sum_{\ell=1}^{j} \epsilon_{\ell} 2^{j-\ell}
$$

associated with the finite subsequence $\left(\epsilon_{\ell}\right)_{\ell=1,2, \ldots, j}$. Clearly, $n_{j}$ is an element of $I_{j}: n_{j}=0$ when " $\epsilon_{\ell}=0$ for all $\ell=1,2, \cdots, j$ ", and $n_{j}=2^{j}-1$ when " $\epsilon_{\ell}=1$ for all $\ell=1,2, \cdots, j "$.

Given an arbitrary infinite sequence $\kappa=\left(\epsilon_{\ell}\right)_{\ell} \in\{0,1\}^{\mathbb{N}}$, the finite subsequence $\left(\epsilon_{\ell}\right)_{\ell=1,2, \ldots, j}$, formed by the $j$ first terms of $\kappa$, defines a unique non negative integer $n_{j} \in I_{j}$ and hence, is associated with the unique wavelet packet located at node $\left(j, n_{j}\right)$ of the decomposition tree. Moreover, this subsequence gives the unique path from $\mathbf{U}^{S}$ to $\mathbf{W}_{j, n_{j}}$ in the wavelet packet tree. Basically, $\left(\epsilon_{\ell}\right)_{\ell=1,2, \ldots, j}$ corresponds to the sequence $H_{\epsilon_{1}}, H_{\epsilon_{2}}, \ldots, H_{\epsilon_{j}}$, of filters successively used to calculate the packet $\mathbf{W}_{j, n_{j}}$.

Conversely, let $n \in I_{j}$. There exists a unique finite sequence $\left(\epsilon_{\ell}\right)_{\ell \in\{1,2, \ldots, j\}}$ of $\{0,1\}^{j}$ such that $n=n_{j}$ where $n_{j}$ is given by (28).

From now on, given an infinite sequence $\kappa$, the natural number $n_{j}=n\left(\kappa_{j}\right)$ and the packet $\mathbf{W}_{j, n_{j}}$ are said to be associated with each other. We also say that the sequences $\kappa$ and $\left(\mathbf{W}_{j, n_{j}}\right)_{j}$ are associated with each other.

Example 1: The following four sequences will often be used in the sequel to illustrate the results we present. These four sequences are

$$
\begin{array}{ll}
\kappa_{0}=(0,0,0,0,0,0, \cdots), & \kappa_{1}=(1,0,0,0,0,0, \cdots), \\
\kappa_{2}=(0,1,0,0,0,0, \cdots), & \kappa_{3}=(0,0,1,0,0,0, \cdots) .
\end{array}
$$

In other words, the general term of the sequence $\kappa_{q}, q=0,1,2$ and 3 , is $\delta[q-\ell]$ for every natural number $\ell$. Clearly, we have that $n_{j}\left(\kappa_{0}\right)=0$ for every natural number $j$. It is also very easy to see that, for $q=1,2,3$, we have $n_{j}\left(\kappa_{q}\right)=0$ for $j=1,2, \ldots, q-1$, and that $n_{j}\left(\kappa_{q}\right)=2^{j-q}$ for $j=q, q+1, \ldots$ 


\subsection{Asymptotic decorrelation with the Shannon wavelet packets}

The QMF $h_{0}^{S}$ and $h_{1}^{S}$ of the Shannon wavelet packet decomposition are the ideal low pass and high pass filters whose Fourier transforms $H_{0}^{S}$ and $H_{1}^{S}$ are:

$$
\left\{\begin{array}{l}
H_{0}^{S}(\omega)=\sqrt{2} \sum_{\ell \in \mathbb{Z}} \chi_{\Delta_{0}}(\omega-2 \pi \ell) \\
H_{1}^{S}(\omega)=\sqrt{2} \sum_{\ell \in \mathbb{Z}} \chi_{\Delta_{1}}(\omega-2 \pi \ell),
\end{array}\right.
$$

where $\Delta_{0}=\left[-\frac{\pi}{2}, \frac{\pi}{2}\right]$, and $\Delta_{1}=\left[-\pi,-\frac{\pi}{2}\right] \cup\left[\frac{\pi}{2}, \pi\right]$. Hereafter, we will use an upper index $S$ in the notations of section 2 when the decomposition is achieved by using the ideal QMF $H_{0}^{S}$ and $H_{1}^{S}$.

Let us define the map $G$ by $G(0)=0$ and the recurrence

$$
\begin{aligned}
& G(2 \ell)=\left\{\begin{array}{cll}
2 G(\ell) & \text { if } G(\ell) & \text { is even } \\
2 G(\ell)+1 & \text { if } G(\ell) & \text { is odd }
\end{array}\right.
\end{aligned}
$$

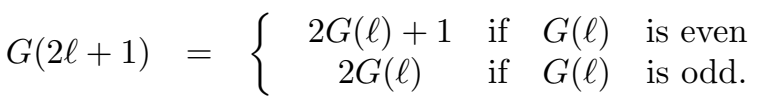

In a more compact form, we can write that

$$
G(2 \ell+\epsilon)=3 G(\ell)+\epsilon-2\left\lfloor\frac{G(\ell)+\epsilon}{2}\right\rfloor,
$$

where $\epsilon \in\{0,1\}$ and $\lfloor z\rfloor$ is the largest integer less than or equal to $z$. The map $G$ is a permutation of $\mathbb{N}$.

For every $(k, \ell) \in \mathbb{N}^{2}$, we define the sets $\Delta_{k, \ell}$ by

$$
\Delta_{k, \ell}=\left[-\frac{(\ell+1) \pi}{2^{k}},-\frac{\ell \pi}{2^{k}}\right] \cup\left[\frac{\ell \pi}{2^{k}}, \frac{(\ell+1) \pi}{2^{k}}\right] .
$$

According to [14, p. 328] (see also [17]), for every $j>0$ and every $n \in I_{j}$, there exists a unique $p=G(n) \in I_{j}$ such that

$$
\left|\widehat{W_{j, n}^{S}}(\omega)\right|=2^{j / 2} \chi_{\Delta_{j, p}}(\omega)
$$

where, like in $(6), W_{j, n}^{S}(t)=2^{-j / 2} W_{n}^{S}\left(2^{-j} t\right), W_{n}^{S}$ stands for the map $W_{n}$ recursively defined by (5) when the pair of QMF is $\left(H_{0}^{S}, H_{1}^{S}\right)$, and $\Delta_{j, p}$ is given by (33). The set $\Delta_{j, p}$ is the so-called subband. The wavelet packet decomposition of a signal of $\mathbf{U}^{S}$, when this decomposition is based on the Shannon QMF, is the ideal subband coding of the signal under consideration. The parameters of each subband can be selected using the integers $j$ and $p$. The first one controls the bandwidth and the second one controls the central frequency.

For every $j>0$, we have $G\left(I_{j}\right)=I_{j}$. Hence, the restriction of $G$ to $I_{j}$ is a permutation of $I_{j}$. This permutation makes it possible to re-order the wavelet packets $W_{j, n}^{S}$ from the lowest to the highest frequency supports. For further details on this re-ordering, the reader may refer to [14, p. 329-330].

Consider the sequence of wavelet packets $\left(\mathbf{W}_{j, n_{j}}^{S}\right)_{j}$ associated with an arbitrary binary sequence $\kappa$ in the sense given in subsection 3.1. At every resolution 
level $j$, it follows from $(34)$ that, $\left|\widehat{W_{j, n_{j}}^{S}}\right|=2^{j / 2} \chi_{\Delta_{j, p_{j}}}$, with

$$
\Delta_{j, p_{j}}=\left[-\frac{\left(p_{j}+1\right) \pi}{2^{j}},-\frac{p_{j} \pi}{2^{j}}\right] \cup\left[\frac{p_{j} \pi}{2^{j}}, \frac{\left(p_{j}+1\right) \pi}{2^{j}}\right]
$$

and $p_{j}=G\left(n_{j}\right)$. When $j$ ranges over $\mathbb{N}$, (35) defines a sequence $\left(\frac{p_{j} \pi}{2^{j}}\right)_{j}$. It is easy to see that this sequence is Cauchy. Indeed, according to (9), we have $n_{j+1}=2 n_{j}+\epsilon$, for some $\epsilon \in\{0,1\}$. Taking (32) into account, we can write that $p_{j+1}=2 p_{j}+\epsilon^{\prime}$, for some $\epsilon^{\prime} \in\{0,1\}$. With an easy recurrence, we obtain

$$
\begin{aligned}
p_{j+q} & =2 p_{j+q-1}+\epsilon_{j+q}^{\prime} \\
& =2^{q} p_{j}+2^{q-1} \epsilon_{j+1}^{\prime}+\cdots+2^{1} \epsilon_{j+q-1}^{\prime}+\epsilon_{j+q}^{\prime} .
\end{aligned}
$$

Therefore,

$$
\frac{p_{j+q} \pi}{2^{j+q}}-\frac{p_{j} \pi}{2^{j}}=\frac{1}{2^{j}} \sum_{\ell=1}^{q} \frac{\epsilon_{\ell+j}^{\prime}}{2^{\ell}} \leq \frac{1}{2^{j}}-\frac{1}{2^{j+q}}<\frac{1}{2^{j}} .
$$

As the sequence $\left(\frac{p_{j} \pi}{2^{j}}\right)_{j}$ is Cauchy, it has a unique limit. This limit

$$
a(\kappa)=\lim _{j \rightarrow+\infty} \frac{p_{j} \pi}{2^{j}}
$$

will play a crucial role in the sequel. Note that $0 \leq a(\kappa) \leq \pi$ for every arbitrary sequence $\kappa$.

Example 1 (continued): Consider the four sequences $k^{q}, q=0,1,2,3$, introduced in example 1 above. For every natural number $j$, we have that $p_{j}\left(\kappa_{0}\right)=G\left(n_{j}\left(\kappa_{0}\right)\right)=0$ since the general term of the sequence $\kappa_{0}$ is zero.

The first $q-1$ terms of the sequence $\kappa_{q}$ when $q=1,2,3$ are also 0 . Therefore, we have $p_{j}\left(\kappa_{q}\right)=G\left(n_{j}\left(\kappa_{q}\right)\right)=0$ for $j=1,2, \ldots, q-1$. Now, an easy recurrence shows that $p_{j}\left(\kappa_{q}\right)=G\left(n_{j}\left(\kappa_{q}\right)\right)=2^{j-q+1}-1$ for $j=q, q+1, \ldots$

As far as the value of $a\left(\kappa_{q}\right)$ is concerned, for $q=0,1,2,3$, we easily see that $\lim _{j \rightarrow \infty} p_{j}\left(\kappa_{0}\right) / 2^{j}=0$, whereas $\lim _{j \rightarrow \infty} p_{j}\left(\kappa_{q}\right) / 2^{j}=1 / 2^{q-1}$ for $q \neq 0$. Therefore, we have $a\left(\kappa_{0}\right)=0$ and $a\left(\kappa_{q}\right)=\pi / 2^{q-1}$ for $q=1,2$ and 3 .

We now state the following result.

Proposition 1 Let $X$ be a second-order random process. Assume that $X$ is zero-mean, WSS, continuous in quadratic mean with PSD $\gamma \in L^{\infty}([-\pi, \pi])$.

Let $\kappa=\left(\epsilon_{k}\right)_{k \in \mathbb{N}}$ be a binary sequence of $\{0,1\}^{\mathbb{N}}$. If $a(\kappa)$ is a continuity point of $\gamma$, then

$$
\lim _{j \rightarrow+\infty} R_{j, n_{j}(\kappa)}^{S}[m]=\gamma(a(\kappa)) \delta[m]
$$

uniformly in $m \in \mathbb{Z}$, and where $R_{j, n_{j}(\kappa)}^{S}$ is the autocorrelation function of the wavelet packet coefficients of $X$ with respect to $\mathbf{W}_{j, n_{j}}^{S}$.

Remark 3 The autocorrelation function $R_{j, n_{j}}^{S}$ of the Shannon wavelet packet coefficients $c_{j, n_{j}}$ derives from (21) and, hence, is given by

$$
R_{j, n_{j}}^{S}[m]=\left.\frac{1}{2 \pi} \int_{\mathbb{R}} \gamma(\omega) \widehat{\mid W_{j, n_{j}}^{S}}(\omega)\right|^{2} e^{i 2^{j} m \omega} d \omega .
$$




\section{Proof: [of proposition 1]}

The function $\gamma$ is even because it is the Fourier transform of the even function $R$. As above, we write $n_{j}$ for $n_{j}(\kappa)$. From (34) and (38), we derive

$$
R_{j, n_{j}}^{S}[m]=\frac{2^{j}}{\pi} \int_{\Delta_{j, p_{j}}^{+}} \gamma(\omega) \cos \left(2^{j} m \omega\right) d \omega
$$

where

$$
\Delta_{j, p_{j}}^{+}=\left[\frac{p_{j} \pi}{2^{j}}, \frac{\left(p_{j}+1\right) \pi}{2^{j}}\right] .
$$

Let $\eta>0$. Since $\gamma$ is continuous at $a(\kappa)$, there exists a positive real number $\alpha>0$, such that, for every $\omega \in[a(\kappa)-\alpha, a(\kappa)+\alpha]$, we have $|\gamma(\omega)-\gamma(a(\kappa))|<\eta$.

In addition, it follows from (36) that

$$
\lim _{j \rightarrow+\infty} p_{j} \pi 2^{-j}=\lim _{j \rightarrow+\infty}\left(p_{j}+1\right) \pi 2^{-j}=a(\kappa),
$$

Thereby, there exists an integer $j_{0}=j_{0}(\alpha)$, such that, for every natural number $j \geq j_{0}$, the values $p_{j} \pi 2^{-j}$ and $\left(p_{j}+1\right) \pi 2^{-j}$ are within the interval $[a(\kappa)-$ $\alpha, a(\kappa)+\alpha]$. It follows that, for every natural number $j \geq j_{0}$ and every $\omega \in \Delta_{j, p_{j}}^{+}$,

$$
|\gamma(\omega)-\gamma(a(\kappa))|<\eta \text {. }
$$

Therefore, for any natural number $j \geq j_{0}$,

$$
\frac{2^{j}}{\pi} \int_{\Delta_{j, p_{j}}^{+}}|\gamma(\omega)-\gamma(a(\kappa))| d \omega<\eta \frac{2^{j}}{\pi} \int_{\Delta_{j, p_{j}}^{+}} d \omega=\eta .
$$

On the other hand, we derive from (39) that for any natural number $j \geq j_{0}$,

$$
\begin{aligned}
\mid R_{j, n_{j}}^{S}[m] & -\frac{2^{j}}{\pi} \int_{\Delta_{j, p_{j}}^{+}} \gamma(a(\kappa)) \cos \left(2^{j} m \omega\right) d \omega \mid \\
& =\left|\frac{2^{j}}{\pi} \int_{\Delta_{j, p_{j}}^{+}}(\gamma(\omega)-\gamma(a(\kappa))) \cos \left(2^{j} m \omega\right) d \omega\right|, \\
& \leq \frac{2^{j}}{\pi} \int_{\Delta_{j, p_{j}}^{+}}|\gamma(\omega)-\gamma(a(\kappa))| d \omega .
\end{aligned}
$$

Hence, we derive from (43) and (44) that, for every natural number $j \geq j_{0}$ and every integer $m$,

$$
\left|R_{j, n_{j}}^{S}[m]-\frac{2^{j}}{\pi} \int_{\Delta_{j, p_{j}}^{+}} \gamma(a(\kappa)) \cos \left(2^{j} m \omega\right) d \omega\right|<\eta .
$$

Since

$$
\frac{2^{j}}{\pi} \int_{\Delta_{j, p_{j}}^{+}} \gamma(a(\kappa)) \cos \left(2^{j} m \omega\right) d \omega=\gamma(a(\kappa)) \delta[m],
$$

we conclude that, for every natural number $j \geq j_{0}$,

$$
\left|R_{j, n_{j}}^{S}[m]-\gamma(a(\kappa)) \delta[m]\right|<\eta
$$

uniformly in $m \in \mathbb{Z}$. 
Remark 4 Proposition 1 shows that the coefficients returned by the Shannon wavelet packet decomposition of process $X$ tend to be decorrelated when the resolution level tends to infinity.

Roughly speaking, the spectral measure of the discrete signal returned in a given subband by the Shannon wavelet packet decomposition of a WSS process tends, with increasing $j$, to $\gamma(a(\kappa))$.

This result also emphasizes the dependence between the autocorrelation function and the binary sequence $\kappa$. For instance, if we consider the four sequences $\kappa_{q}, q=0,1,2,3$ of example 1 , we have

$$
\lim _{j \rightarrow+\infty} R_{j, n_{j}\left(\kappa_{q}\right)}^{S}[m]=\gamma\left(\pi / 2^{q-1}\right) \delta[m]
$$

for $q=1,2$ and 3 , whereas

$$
\lim _{j \rightarrow+\infty} R_{j, n_{j}\left(\kappa_{0}\right)}^{S}[m]=\gamma(0) \delta[m] .
$$

Remark 5 (On the speed of the decorrelation process) Let $\omega_{0}>0$ and

$$
\gamma(\omega)=\left(1-\frac{|\omega|}{\omega_{0}}\right) \chi_{[-\pi, \pi] \cap\left[-\omega_{0}, \omega_{0}\right]}(\omega) .
$$

We assume that $\gamma$ represents the PSD of some zero-mean WSS random process. We have

$$
R_{j, n_{j}}^{S}[m]=\frac{2^{j}}{\pi} \int_{\Delta_{j, p_{j}}^{+} \cap\left[0, \omega_{0}\right]}\left(1-\frac{\omega}{\omega_{0}}\right) \cos \left(2^{j} m \omega\right) d \omega .
$$

If $\omega_{0}>\pi, \Delta_{j, p_{j}}^{+} \cap\left[0, \omega_{0}\right]=\Delta_{j, p_{j}}^{+}$, and we obtain that

$$
R_{j, n_{j}}^{S}[m]=\left\{\begin{array}{cc}
1-\frac{\pi}{\omega_{0}} \frac{p_{j}}{2^{j}}-\frac{\pi}{\omega_{0}} \frac{1}{2^{j+1}} \quad \text { if } m=0 \\
\frac{(-1)^{m p_{j}}-(-1)^{m\left(p_{j}+1\right)}}{\pi \omega_{0} m^{2} 2^{j}} & \text { if } m \neq 0,
\end{array}\right.
$$

and thus,

$$
\left|R_{j, n_{j}}^{S}[m]-\gamma\left(\frac{\pi p_{j}}{2^{j}}\right) \delta[m]\right| \leq \frac{\pi}{\omega_{0}} \frac{1}{2^{j+1}} .
$$

It follows that $\gamma\left(\pi p_{j} / 2^{j}\right) \delta[m]$ is an approximation of $R_{j, n_{j}}^{S}[m]$ with a margin of $\pi / \omega_{0} 2^{j+1}$. This highlights the speed of the decorrelation process for $\omega_{0}>\pi$.

If $\omega_{0} \leq \pi$, then the function $\gamma$ is null on $\left[\omega_{0}, \pi\right]$. If $a(\kappa)$ is such that $\omega_{0}<$ $a(\kappa) \leq \pi$, then there exists $\alpha>0$ such that $\gamma$ is null on $] a(\kappa)-\alpha, a(\kappa)+\alpha[$ and there exists $j_{1}$ such that for all $\left.j \geq j_{1}, \Delta_{j, p_{j}}^{+} \subset\right] a(\kappa)-\alpha, a(\kappa)+\alpha[$. Thus, the autocorrelation $R_{j, n_{j}}^{S}[m]$ is null for all $j \geq j_{1}$. Now, consider that $0 \leq a(\kappa)<$ $\omega_{0}$. Then for $j$ greater than or equal to a certain $j_{2}, \Delta_{j, p_{j}}^{+} \subset\left[0, \omega_{0}\right]$. Thus, for all $j \geq j_{2}, \Delta_{j, p_{j}}^{+} \cap\left[0, \omega_{0}\right]=\Delta_{j, p_{j}}^{+}$and we obtain that (53) holds true. It is also easy to see that for $j$ greater than or equal to a certain $j_{3}$, (53) holds true when $a(\kappa)=\omega_{0}$. It follows that if $\omega_{0} \leq \pi$, there exists $j_{0}$ such that for all $j \geq j_{0}$, (53) holds true.

More generally, consider an arbitrary PSD $\gamma$ with support in $[-\pi, \pi]$. When the resolution level $j$ is sufficiently large, $\gamma$ can be seen as a linear function 
in $\Delta_{j, p_{j}}^{+}$. Then, we can generalise the above result, saying that there exists a finite level $j_{0}$ such that for any resolution level $j \geq j_{0}, R_{j, n_{j}}^{S}[\mathrm{~m}]$ is appreciatively equivalent to $\gamma\left(\pi p_{j} / 2^{j}\right) \delta[m]$ with a margin of $A / 2^{j}$. The constant $A$ and the level $j_{0}$ depend on the shape of $\gamma$ on $\Delta_{j, p_{j}}^{+}$.

\subsection{Asymptotic decorrelation with non-ideal QMF}

We consider QMF $\left(h_{0}^{[r]}, h_{1}^{[r]}\right)$ that depend on a parameter $r$ such that, for $\epsilon \epsilon$ $\{0,1\}$,

$$
\lim _{r \rightarrow \infty} H_{\epsilon}^{[r]}=H_{\epsilon}^{S} \quad \text { (a.e), }
$$

where $H_{0}^{[r]}$ and $H_{1}^{[r]}$ are the Fourier transforms of $h_{0}^{[r]}$ and $h_{1}^{[r]}$ respectively, and where, as above, $H_{0}^{S}$ and $H_{1}^{S}$ are the Fourier transforms of the Shannon QMF. We assume that $r$ is an integer. As mentioned in the introduction, this parameter is called the order of the QMF $H_{0}^{[r]}$ and $H_{1}^{[r]}$. It has different meanings for the Daubechies and the Battle-Lemarié QMF.

According to $[18,19,20]$, the Daubechies QMF satisfy (54), the order $r$ of a given pair $\left(h_{0}^{[r]}, h_{1}^{[r]}\right)$ of such QMF being the number of vanishing moments of the associated Daubechies wavelet function. The order $r$ is also the zero multiplicity at $\pi$ of $H_{0}^{[r]}$.

It follows from [21] that the Battle-Lemarié filters satisfy (54) as well; the order $r$ of a given pair $\left(h_{0}^{[r]}, h_{1}^{[r]}\right)$ of such QMF is the spline order of the scaling function associated with $H_{0}^{[r]}$.

Theorem 1 Let $X$ be a second-order random process that satisfies the assumptions of proposition 1. Let $\kappa=\left(\epsilon_{k}\right)_{k \in \mathbb{N}}$ be a binary sequence of $\{0,1\}^{\mathbb{N}}$. Consider, for every $r$, the sequence of wavelet packets $\left(\mathbf{W}_{j, n_{j}(\kappa)}^{[r]}\right)_{j \geq 0}$ associated with $\kappa$, where the decomposition of $\mathbf{U}^{S}$ is achieved by the $Q M F\left(h_{0}^{[r]}, h_{1}^{[r]}\right)$.

For every given natural number $j$, let $R_{j, n_{j}(\kappa)}^{[r]}$ stands for the autocorrelation function of $X$ with respect to the packet $\mathbf{W}_{j, n_{j}(\kappa)}^{[r]}$.

i) We have

$$
\lim _{r \rightarrow+\infty} R_{j, n_{j}}^{[r]}[m]=R_{j, n_{j}}^{S}[m],
$$

and this convergence is uniform in $m \in \mathbb{Z}$.

ii) In addition, if $\gamma$ is continuous at $a(\kappa)$, then,

$$
\lim _{j \rightarrow+\infty}\left(\lim _{r \rightarrow+\infty} R_{j, n_{j}(\kappa)}^{[r]}[m]\right)=\gamma(a(\kappa)) \delta[m]
$$

uniformly in $m \in \mathbb{Z}$.

Remark 6 According to theorem 1 and if $\gamma$ is continuous at a( $\kappa)$, then, for every given real number $\eta>0$, there exists a natural number $j_{0}$ with the following 
property: For every $j \geq j_{0}$, there exists $r_{0}=r_{0}\left(j, n_{j}\right)>0$ such that, for every $r \geq r_{0}$ and every $m \in \mathbb{Z}$,

$$
\left|R_{j, n_{j}(\kappa)}^{[r]}[m]-\gamma(a(\kappa)) \delta[m]\right|<\eta .
$$

Thus, according to (57) we obtain nearly-white DWPT coefficients (with a margin of $\eta$ ) at resolution level $j_{0}$ and by using $Q M F$ with order $r_{0}$.

\section{Proof: [of theorem 1]}

As above, we set $n_{j}=n_{j}(\kappa)$. The autocorrelation function $R_{j, n_{j}}^{[r]}$ derives from (21) and is equal to

$$
R_{j, n_{j}}^{[r]}[m]=\left.\frac{1}{2 \pi} \int_{\mathbb{R}} \gamma(\omega) \widehat{\mid W_{j, n_{j}}^{[r]}}(\omega)\right|^{2} e^{i 2^{j} m \omega} d \omega .
$$

For every $m \in \mathbb{Z}$, we have

$$
\begin{aligned}
& R_{j, n_{j}}^{[r]}[m]-R_{j, n_{j}}^{S}[m] \mid \\
& \leq \quad \frac{1}{2 \pi} \int_{\mathbb{R}}|\gamma(\omega)|\left|\widehat{W_{j, n_{j}}^{[r]}}(\omega)\right|^{2}-\left.\widehat{W_{j, n_{j}}^{S}}(\omega)\right|^{2} \mid d \omega
\end{aligned}
$$

But from appendix E, we have

$$
\widehat{W_{n_{j}}^{S}}(\omega)=\left[\prod_{\ell=1}^{j} H_{\epsilon_{\ell}}^{S}\left(\frac{\omega}{2^{j+1-\ell}}\right)\right] \widehat{\Phi^{S}}\left(\frac{\omega}{2^{j}}\right)
$$

and

$$
\widehat{W_{n_{j}}^{[r]}}(\omega)=\left[\prod_{\ell=1}^{j} H_{\epsilon_{\ell}}^{[r]}\left(\frac{\omega}{2^{j+1-\ell}}\right)\right] \widehat{\Phi^{S}}\left(\frac{\omega}{2^{j}}\right) .
$$

Thus, from (6), (54), (60) and (61), and taking into acount that $\left|H_{\epsilon_{\ell}}^{[r]}(\omega)\right|$ and $\left|H_{\epsilon_{\ell}}^{S}(\omega)\right|$ are less than or equal to 1 , we obtain

$$
\lim _{r \rightarrow+\infty}\left|\widehat{W_{j, n_{j}}^{[r]}}\right|^{2}=\left.\widehat{\mid W_{j, n_{j}}^{S}}\right|^{2} \quad \text { (a.e), }
$$

and

$$
\left|\widehat{\mid W_{j, n_{j}}^{[r]}}(\omega)\right|^{2}-\left.\left.\widehat{W_{j, n_{j}}^{S}}(\omega)\right|^{2}\left|\leq 2^{j+1}\right| \widehat{\Phi^{S}}(\omega)\right|^{2} .
$$

Statement i) follows from (59), (62), (63), and Lebesgue's dominated convergence theorem.

We just obtain statement ii) from proposition 1 and statement i).

\section{Experimental results}

The theoretical results presented above are of asymptotic nature. The role of this section is to reflect the manner in which the decorrelation appears in practice when the resolution level and the order of the filters are finite.

Consider a random process satisfying the assumptions of theorem 1 . With the same notations as those used above, a consequence of theorem 1 is the 
existence of some resolution level $j$ and some order $r$ such that (57) holds true when the wavelet packet decomposition is initialised with the samples of the process. Equation (56) also tells us that the value of the autocorrelation function at lag 0 of the wavelet packet coefficients tends to the value of the PSD at a specific point that depends on the path followed in the wavelet packet tree. The following experimental results are aimed at illustrating these two facts.

We carried out experimental tests where the samples $(X(k))_{k}$ of process $X$ at the input of the wavelet packet decomposition form a discrete Auto-Regressive (AR) process. This AR process is such that $X(k)=\alpha X(k-1)+W(k)$, where $0<\alpha<1$, and $k=1,2, \ldots, 2^{20}$. The random variables $(W(k))_{k}$ are Gaussian, independent and identically distributed with zero-mean and unitary standarddeviation. The samples $(X(k))_{k}$ were generated by filtering the discrete random process $(W(k))_{k}$ through the discrete AR filter whose transfer function is $(1-$ $\alpha) /\left(1-\alpha z^{-1}\right)$. We present the results obtained with $\alpha=0.9$. The corresponding PSD is displayed in figure 2, and the autocorrelation is displayed in figure 3.

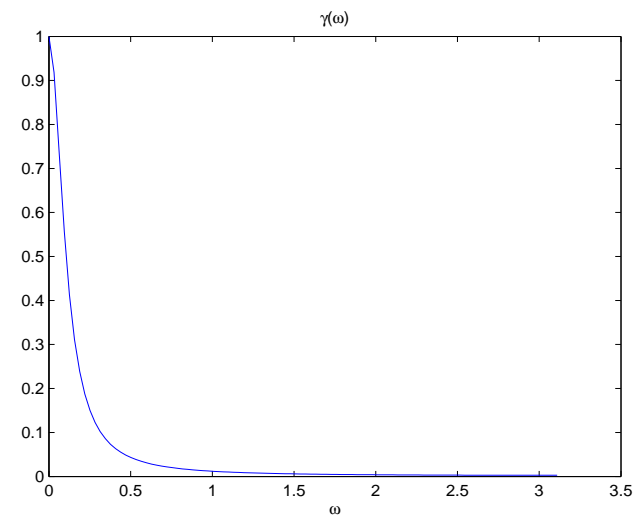

Figure 2: PSD of the random process used to carried out experimental tests. These random processes were synthesized by filtering some white noise with a first order AR filter.

The experimental results detailed below show that, for the AR process considered above, the asymptotic decorrelation stated by theorem 1 can actually be attained with reasonable values for the resolution level $j$ and the order $r$ of the filters. These experimental results have been obtained by achieving full wavelet packet decompositions of 100 realizations of the AR random process specified above. The decompositions are performed by using Daubechies filters and the results given hereafter are average values over these 100 realizations.

We begin with experimental results illustrating the influence of the resolution level and the order of the QMF on the decorrelation process. We then address the convergence of the autocorrelation functions of the wavelet packet coefficients at lag 0 . 


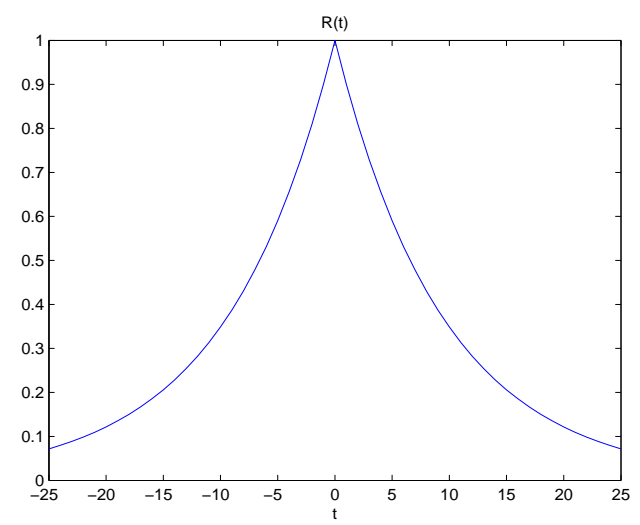

Figure 3: Autocorrelation function of the random process whose PSD is plotted in figure 2 .

\subsection{Influence of the resolution level and the order of the filters on the decorrelation process}

The full wavelet packet decompositions are now achieved when the QMF are the Daubechies filters of orders $1,2, \ldots, 20$. The average empirical autocorrelation functions of the wavelet packet coefficients $c_{j, n_{j}\left(\kappa_{q}\right)}$ for $q=0,1,2,3$, (see example 1) are calculated on the basis of 1500 coefficients per packet $\mathbf{W}_{j, n_{j}\left(\kappa_{q}\right)}$, which is possible for every decomposition level considered below since we have $2^{20}$ input samples.

It turns out that the wavelet packet coefficients can be considered as significantly decorrelated when the resolution level is 6 and the order of the QMF is greater than, or equals 7 . For instance, figure 4 shows the empirical normalized autocorrelation functions at resolution levels 3 and 6 , when $r$ equals 1 and 7 for the AR process. These autocorrelation functions are normalized in order to appreciate the gain in decorrelation. These figures underline the importance of the role played by the resolution level and the order of the QMF.

The importance of the resolution level can be appreciated by noting that the coefficients at resolution level 6 are less correlated than those obtained at level 3 (compare the first and the second columns of figure 4).

The role played by the QMF order is the following. At level 6 and for every wavelet packet, the decomposition based on the Daubechies QMF with order 7 yields better decorrelation than that obtained by the decomposition based on the Daubechies QMF of order 1 (in figure 4, compare the second and the third columns).

\subsection{The limit value of the correlation function at lag 0}

Consider the sequence $\left(R_{j, n_{j}}^{[r]}\right)_{j}$ where, for every natural number $j, R_{j, n_{j}}^{[r]}$ is the autocorrelation function of the wavelet packet coefficients associated with $X$ with respect to the sequence $\left(\mathbf{W}_{j, n_{j}}^{[r]}\right)_{j}$. According to theorem 1 , the value $R_{j, n_{j}}^{[r]}[m]$ must be close to $\gamma(a(\kappa)) \delta[m]$ if $j$ and $r$ are both large enough, $r$ being above a value that depends on $j$. We recall that $\gamma(a(\kappa))$ is the value of the PSD 
of the random process $X$ at $a(\kappa)$ where $a(\kappa)$ is given by (36) and $\kappa$ is the binary sequence associated with $\left(\mathbf{W}_{j, n_{j}}^{[r]}\right)_{j}$.

Let us illustrate this result for the value of the autocorrelation function at lag 0 and the binary sequences $\kappa_{0}, \kappa_{1}, \kappa_{2}$, and $\kappa_{3}$, introduced in example 1 . We focus on the Daubechies QMF with order $r=7$.

We consider again the AR random process introduced above and whose PSD is that of figure 2. We observe that, quite rapidly, the terms of the sequence $\left(R_{j, n_{j}}^{[7]}[0]\right)_{j}$ become close to $\gamma(a(\kappa))$ when $j$ increases. This is shown in figure 5. This figure displays the average values $R_{j, n_{j}\left(\kappa_{q}\right)}^{[7]}[0]$ for $j=0,1,2, \cdots, 9$ and $q=0,1,2,3$. We plot the limit value $\gamma(a(\kappa))$ in dotted line in these figures. We see that, as the resolution level $j$ increases up to 6 , the value at 0 of the autocorrelation function of the wavelet packet coefficients associated with each of the four sequences is sufficiently close to the value of the PSD at $a(\kappa)$.

In order to specify the accuracy of the results of figure 5 , the following table presents the average values $R_{j, n_{j}\left(\kappa_{q}\right)}^{[7]}[0]$ for $j=6,7,8,9$, as well as their $95 \%$ confidence intervals calculated via the standard t-test. Compare these average values with the exact values of $\gamma\left(a\left(\kappa_{q}\right)\right)$ that can easily be computed for the AR process considered in this section. We have $\gamma\left(a\left(\kappa_{0}\right)\right)=1, \gamma\left(a\left(\kappa_{1}\right)\right)=0.002770$, $\gamma\left(a\left(\kappa_{2}\right)\right)=0.005703$ and $\gamma\left(a\left(\kappa_{3}\right)\right)=0.020086$.

\begin{tabular}{|c||c|c|}
\hline Level $(j)$ & 6 & 7 \\
\hline \hline$R_{j, n_{j}\left(\kappa_{0}\right)}^{[7]}(0)$ & $0.9313 \pm 0.0074$ & $0.9810 \pm 0.0075$ \\
$R_{j, n_{j}\left(\kappa_{1}\right)}^{[7}(0)$ & $0.002777 \pm 0.000021$ & $0.002778 \pm 0.000022$ \\
$R_{j, n_{j}\left(\kappa_{2}\right)}^{[7]}(0)$ & $0.005587 \pm 0.000041$ & $0.005591 \pm 0.000041$ \\
$R_{j, n_{j}\left(\kappa_{3}\right)}^{[7]}(0)$ & $0.01887 \pm 0.00012$ & $0.01862 \pm 0.00012$ \\
\hline \hline \hline Level $(j)$ & 8 & 9 \\
\hline \hline$R_{j, n_{j}\left(\kappa_{0}\right)}^{[7]}(0)$ & $0.9957 \pm 0.0075$ & $0.9938 \pm 0.0061$ \\
$R_{j, n_{j}\left(\kappa_{1}\right)}^{[70)}$ & $0.002779 \pm 0.000020$ & $0.002776 \pm 0.000019$ \\
$R_{j, n_{j}\left(\kappa_{2}\right)}^{[70)}$ & $0.005602 \pm 0.000042$ & $0.005555 \pm 0.000041$ \\
$R_{j, n_{j}\left(\kappa_{3}\right)}^{[7]}(0)$ & $0.01849 \pm 0.00012$ & $0.01863 \pm 0.00012$ \\
\hline
\end{tabular}

Table 1: Average values and associated $95 \%$ confidence intervals for $R_{j, n_{j}\left(\kappa_{q}\right)}^{[7]}[0]$ computed over 100 realizations of the process whose PSD is that of figure 2 .

To conclude this section, note that the AR process used to illustrate the decorrelation process is chosen so that the slope of its PSD is quite sharp at $\omega=0$. According to remark 5 , this sharpness slows down the DWPT whitening effect stated by theorem 1. In fact, the flatter the PSD, the faster the decorrelation is achieved by increasing the decomposition level and the QMF order. In particular, the foregoing experimental results are even better for an AR process $X(k)=\alpha X(k-1)+W(k)$ with $0<\alpha<0.9$. 

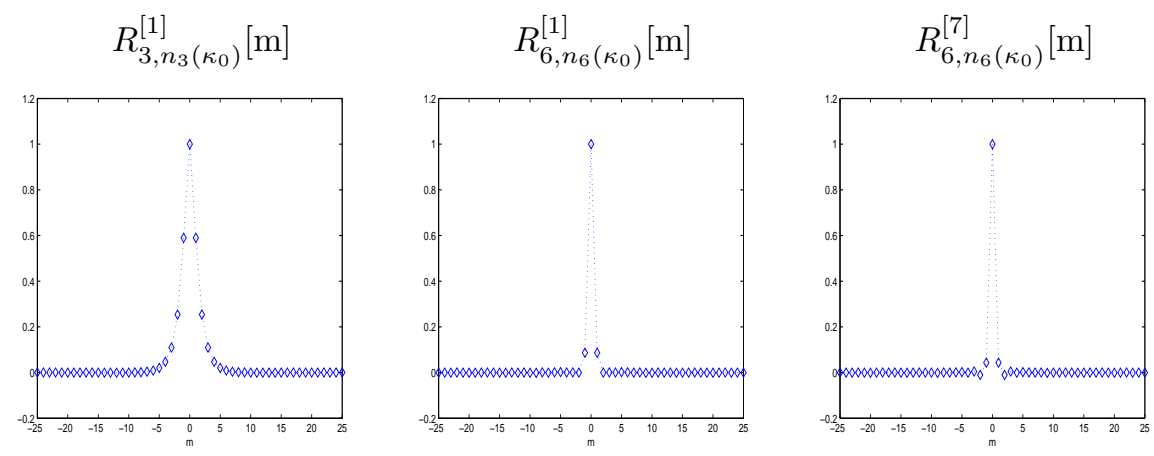

$R_{3, n_{3}\left(\kappa_{1}\right)}^{[1]}[\mathrm{m}]$

$R_{6, n_{6}\left(\kappa_{1}\right)}^{[1]}[\mathrm{m}]$

$R_{6, n_{6}\left(\kappa_{1}\right)}^{[7]}[\mathrm{m}]$
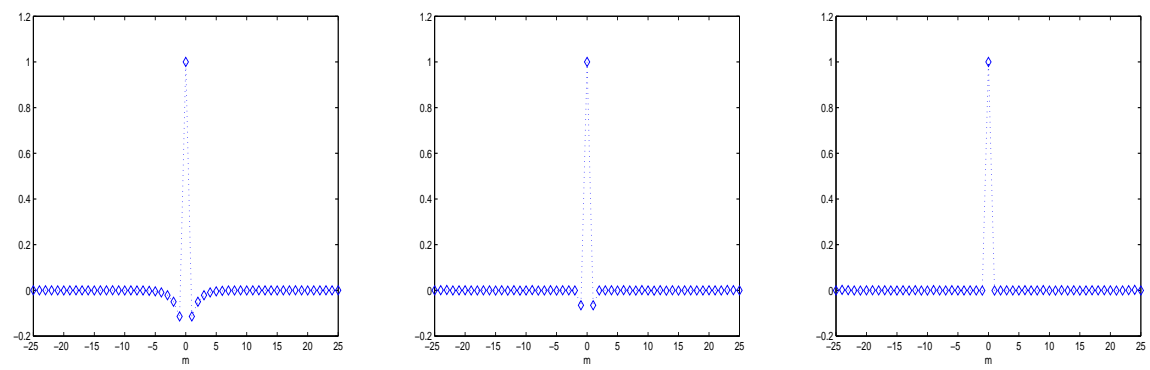

$R_{3, n_{3}\left(\kappa_{2}\right)}^{[1]}[\mathrm{m}]$

$R_{6, n_{6}\left(\kappa_{2}\right)}^{[1]}[\mathrm{m}]$

$R_{6, n_{6}\left(\kappa_{2}\right)}^{[7]}[\mathrm{m}]$
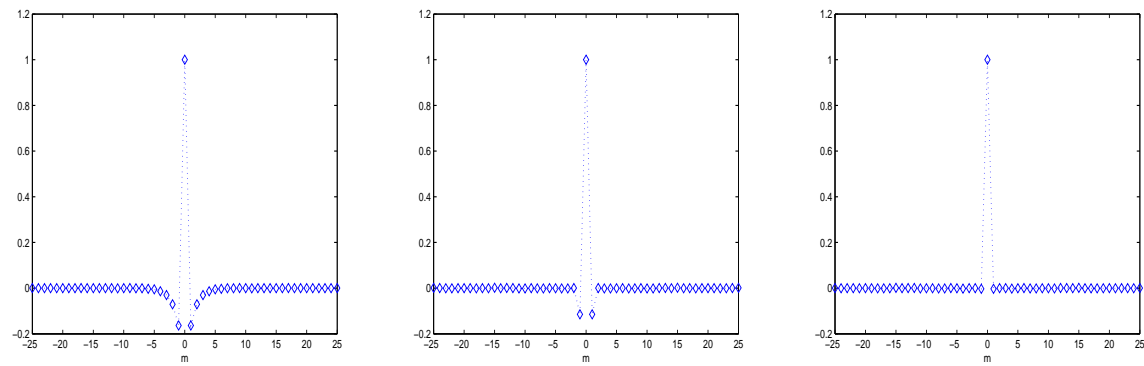

$R_{3, n_{3}\left(\kappa_{3}\right)}^{[1]}[\mathrm{m}]$

$R_{6, n_{6}\left(\kappa_{3}\right)}^{[1]}[\mathrm{m}]$

$R_{6, n_{6}\left(\kappa_{3}\right)}^{[7]}[\mathrm{m}]$
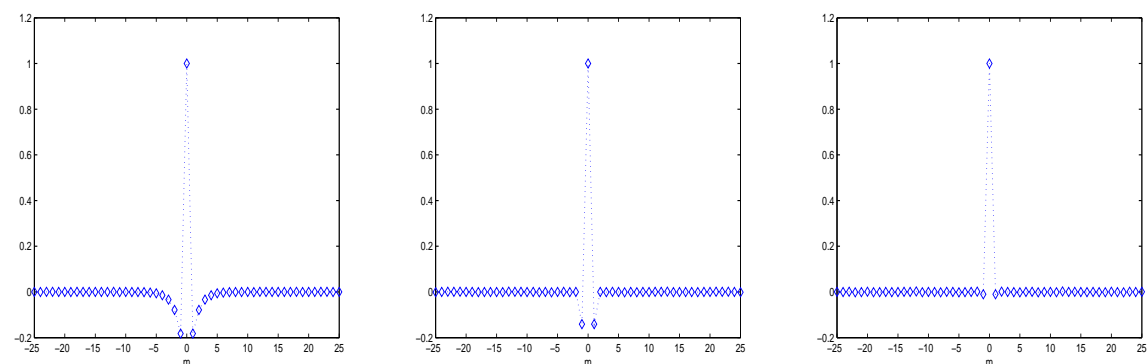

Figure 4: Some normalized autocorrelation functions of the wavelet packet coefficients returned for the 4 sequences of example 1, at decomposition levels 3 and 6. The decompositions concern the random process with PSD given by figure 2. They are achieved by using the Daubechies filters of orders 1 and 7 . 


$$
R_{j, n_{j}\left(\kappa_{0}\right)}^{[7]}[0]
$$

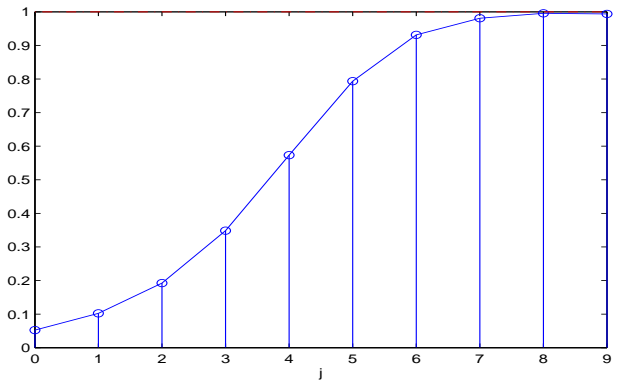

$$
R_{j, n_{j}\left(\kappa_{1}\right)}^{[7]}[0]
$$

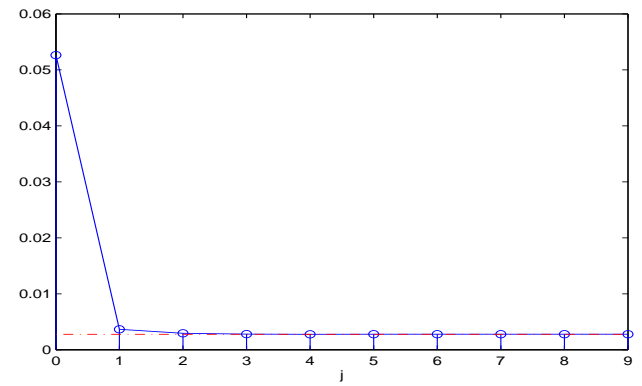

$$
R_{j, n_{j}\left(\kappa_{2}\right)}^{[7]}[0]
$$

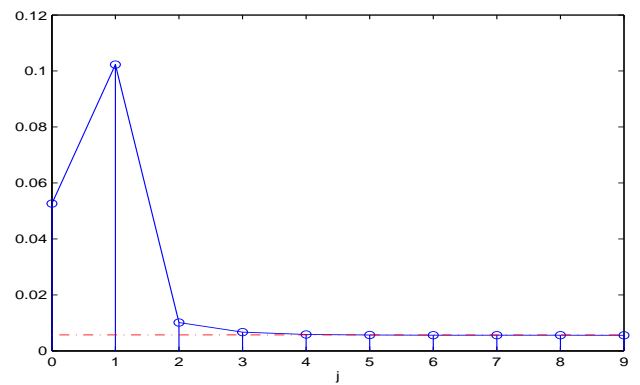

$$
R_{j, n_{j}\left(\kappa_{3}\right)}^{[7]}[0]
$$

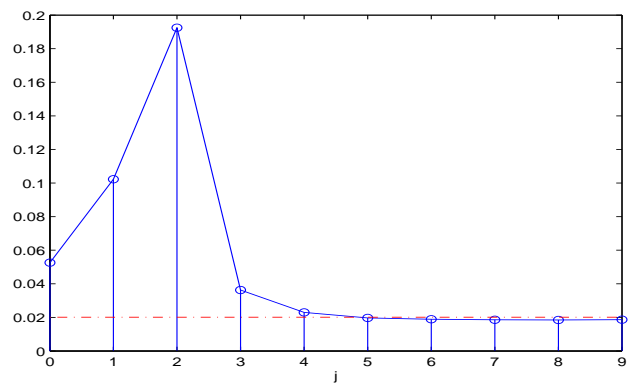

Figure 5: Convergence of $R_{j, n_{j}\left(\kappa_{q}\right)}^{[7]}[0]$ to $\gamma\left(a\left(\kappa_{q}\right)\right)$ with $j$ for the four test sequences. The decomposition concerns the random process whose PSD is that of figure 2, and has been achieved by using the Daubechies filters of order 7 . 


\section{Conclusion}

This paper provides further details concerning the asymptotic decorrelation of the wavelet packet coefficients resulting from the decomposition of a bandlimited WSS random process. By choosing a sufficiently large resolution level and, then, by increasing the order of the filters with respect to the chosen resolution level, the wavelet packet coefficients tend to become decorrelated. The results presented in this paper complement those established in [10], [11] and [15].

Figure 4 highlights the quality of the decorrelation process. This quality can be regarded as rather good: compare the autocorrelation functions of the input WSS process (figure 3) to the autocorrelation functions of the associated coefficients (figure 4). The performance of this experiment can be improved by increasing the order of the QMF (compare the second and the third column of figure 4). These results suggest using a full wavelet packet decomposition with six or more resolution levels and Daubechies filters with seven or more vanishing moments. The experimental results presented in this paper indicate that the asymptotic decorrelation can be approximately attained in practice with reasonable values for the resolution level and the order of the QMF. This relates to the convergence speed of the Daubechies QMF to the Shannon filters (see figure 6) and also to the speed of the decorrelation process (see remark 5).

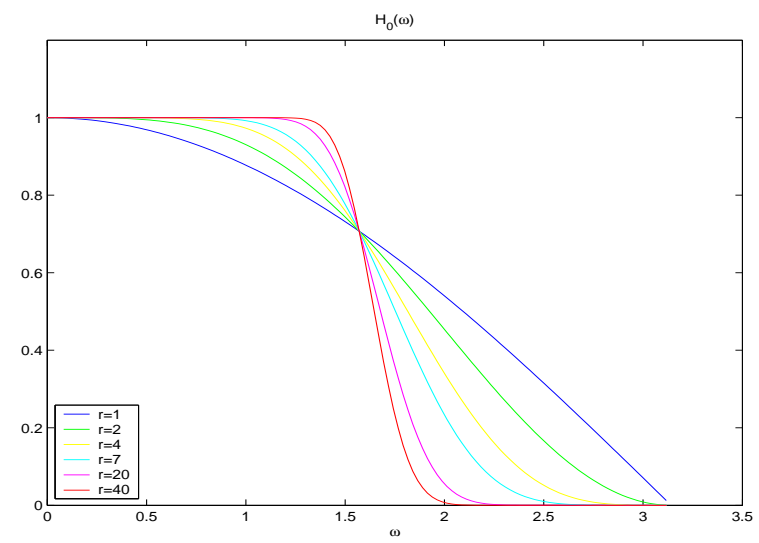

Figure 6: Magnitude response of some Daubechies scaling filters. This figure illustrates the convergence speed of the Daubechies QMF to the Shannon filters. It displays the magnitude response of the Daubechies scaling filters with orders $r=1,2,4,7,20$, and 40 . This scaling filter is obtained by using the MATLAB routine dbaux of the wavelet toolbox.

Various applications of these results are thinkable. Indeed, many basic models and techniques employed in signal processing and statistics are valid, or significantly much simpler and efficient, when data are uncorrelated. For instance, we can think of immediate applications in spectral analysis, but also in denoising and deconvolution. In particular, the authors' feeling is that the result of the present paper can contribute to the study of non-uniform sampling or local analysis of the PSD of a signal [22], [23]. The analysis of the variance of the wavelet packets in order to detect change-points, [24], seems to be an- 
other application of interest. Theorem 1 also suggests extending Donoho and Johnstone's approach [25], [26] to the denoising of signals corrupted by coloured noise. The idea would be to determine a wavelet packet basis that decorrelates sufficiently well the input noise. In each packet, noise could then be removed by applying a suitable thresholding function whose threshold height is a function of the variance of the noise coefficients. Denoising and deconvolution being very similar problems [14], [27], the approach described just above could possibly be extended to deconvolution.

In future work, we plan to investigate how the theoretical results presented above apply to telecommunication systems where wavelets are gaining more and more interest (see [28], [29], [30], [31], among others). In particular, it seems relevant to study to what extent the results of this paper extend to nonstationary or almost cyclostationary processes and relate to properties such as those given in [32].

\section{Acknowledgement}

The authors thank the reviewers for their suggestions concerning applications of the results presented in this paper and the organization of section 4 . The authors are also very grateful to Janet Ormrod who improved the English of this paper.

A

According to Loeve's lemma ([33]), and for $-\infty<a \leq b<\infty$, the stochastic integral

$$
\int_{a}^{b} X(t) W_{j, n, k}(t) d t
$$

is well defined if, and only if

$$
\mathbb{E}\left[\int_{a}^{b} X(t) W_{j, n, k}(t) d t \int_{a}^{b} \overline{X(s) W_{j, n, k}(s)} d s\right]
$$

is well defined, i.e. if, and only if the Riemann integral

$$
\int_{a}^{b} \int_{a}^{b} R(t, s) W_{j, n, k}(t) W_{j, n, k}(s) d t d s
$$

exists and is finite. We can then define the stochastic integral

$$
\int_{\mathbb{R}} X(t) W_{j, n, k}(t) d t
$$

as the limit of $\int_{a}^{b} X(t) W_{j, n, k}(t) d t$ (with convergence in quadratic mean) when $a \rightarrow-\infty$ and $b \rightarrow+\infty$, and this, provided that the integral

$$
\iint_{\mathbb{R}^{2}} R(t, s) W_{j, n, k}(t) W_{j, n, k}(s) d t d s
$$

exists. This is the case if we suppose that $W_{n}$ is compactly supported or has a sufficiently fast decay (see [6] among others). Given a resolution level $j$ and 
$n \in I_{j}$ (so, given $\mathbf{W}_{j, n}$ ), the wavelet packet decomposition of $X$ returns the random variables

$$
c_{j, n}[k]=\int_{\mathbb{R}} X(t) W_{j, n, k}(t) d t, \quad k \in \mathbb{Z} .
$$

\section{B}

According to (5), (6) and (7), and for $\epsilon \in\{0,1\}$, we have

$$
\begin{aligned}
W_{j+1,2 n+\epsilon, k}(t) & =\tau_{2^{j+1} k} W_{j+1,2 n+\epsilon}(t) \\
& =\tau_{2^{j+1} k} 2^{-(j+1) / 2} W_{2 n+\epsilon}\left(2^{-(j+1)} t\right) \\
& =\tau_{2^{j+1} k} 2^{-(j+1) / 2} \sqrt{2} \sum_{\ell \in \mathbb{Z}} h_{\epsilon}[\ell] W_{n}\left(2^{-j} t-\ell\right) \\
& =\tau_{2^{j+1} k} 2^{-j / 2} \sum_{\ell \in \mathbb{Z}} h_{\epsilon}[\ell] W_{n}\left(2^{-j} t-\ell\right) \\
& =2^{-j / 2} \sum_{\ell \in \mathbb{Z}} h_{\epsilon}[\ell] W_{n}\left(2^{-j} t-2 k-\ell\right) \\
& =2^{-j / 2} \sum_{\ell \in \mathbb{Z}} h_{\epsilon}[\ell-2 k] W_{n}\left(2^{-j} t-\ell\right) \\
& =\sum_{\ell \in \mathbb{Z}} h_{\epsilon}[\ell-2 k] W_{j, n, \ell}(t),
\end{aligned}
$$

and thus $W_{j+1,2 n+\epsilon, k}=\sum_{\ell \in \mathbb{Z}} h_{\epsilon}[\ell-2 k] W_{j, n, \ell}$. The equalities above must be understood in the quadratic sense. If the impulse response of the filters have finite length, these equalities hold true pointwise.

C

With the notations of appendix A, we assume that the mean-square integral (64) is well defined. The autocorrelation function $R_{j, n}$ of the discrete random process $c_{j, n}$ is defined by

$$
R_{j, n}[k, \ell]=\iint_{\mathbb{R}^{2}} R(t-s) W_{j, n, k}(t) W_{j, n, \ell}(s) d t d s,
$$

which can be written in the following form

$$
R_{j, n}[k, \ell]=\iint_{\mathbb{R}^{2}} R(t) W_{j, n, k}(t+s) W_{j, n, \ell}(s) d t d s .
$$

In addition, since $W_{j, n, k}(t)=2^{-j / 2} W_{n}\left(2^{-j} t-k\right)$, we have

$$
\begin{aligned}
\int_{\mathbb{R}} W_{j, n, k}(t+s) W_{j, n, \ell}(s) d s & =\int_{\mathbb{R}} W_{n}\left(s+2^{-j} t-k\right) W_{n}(s-\ell) d s, \\
& =\left\langle\tau_{k-\ell-2^{-j} t} W_{n}, W_{n}\right\rangle_{L^{2}(\mathbb{R})}, \\
& =\left.\frac{1}{2 \pi} \int_{\mathbb{R}} \widehat{W_{n}}(\omega)\right|^{2} e^{i\left(k-\ell-2^{-j} t\right) \omega} d \omega,
\end{aligned}
$$


where we use the Parseval formula to obtain the last equality. We thus have

$$
R_{j, n}[k, \ell]=\frac{1}{2 \pi} \iint_{\mathbb{R}^{2}} R(t)\left|\widehat{W_{n}}(\omega)\right|^{2} e^{i\left(k-\ell-2^{-j} t\right) \omega} d t d \omega .
$$

And since

$$
\int_{\mathbb{R}} R(t) e^{-i 2^{-j} t \omega} d t=\gamma\left(\frac{\omega}{2^{j}}\right),
$$

we derive that

$$
R_{j, n}[k, \ell]=\frac{1}{2 \pi} \int_{\mathbb{R}} \gamma\left(\frac{\omega}{2^{j}}\right)\left|\widehat{W_{n}}(\omega)\right|^{2} e^{i(k-\ell) \omega} d \omega .
$$

D

Let $Z$ be the orthogonal stochastic measure, associated with the spectral measure $m$ of the continuous and second order WSS random process $X$ (WienerKhintchine's theorem). We have

$$
m(d \omega)=\frac{1}{2 \pi} \gamma(\omega) d \omega,
$$

where $\gamma$ is the PSD of $X, \gamma$ has support in $[-\pi, \pi]$. Then, we can write $X(t)$ as a stochastic integral :

$$
X(t)=\int_{\mathbb{R}} e^{i t \omega} Z(d \omega) .
$$

Now, according to [34, lemma 5, p. 197-198], we have

$$
\begin{aligned}
\int_{\mathbb{R}} X(t) \Phi^{S}(t-k) d t & =\int_{\mathbb{R}}\left(\int_{\mathbb{R}} e^{i t \omega} Z(d \omega)\right) \Phi^{S}(t-k) d t, \\
& =\int_{\mathbb{R}}\left(\int_{\mathbb{R}} \Phi^{S}(t-k) e^{i t \omega} d t\right) Z(d \omega), \\
& =\int_{\mathbb{R}} \widehat{\Phi^{S}}(\omega) e^{i k \omega} Z(d \omega) .
\end{aligned}
$$

We obtain

$$
X(k)-\int_{\mathbb{R}} X(t) \Phi^{S}(t-k) d t=\int_{\mathbb{R}} e^{i k \omega}\left(1-\widehat{\Phi^{S}}(\omega)\right) Z(d \omega),
$$

and then,

$$
\begin{aligned}
& \mathbb{E}\left[\left|X(k)-\int_{\mathbb{R}} X(t) \Phi^{S}(t-k) d t\right|^{2}\right] \\
&=\int_{\mathbb{R}}\left|1-\widehat{\Phi^{S}}(\omega)\right|^{2} m(d \omega) . \\
&=\frac{1}{2 \pi} \int_{-\pi}^{\pi}\left|1-\widehat{\Phi^{S}}(\omega)\right|^{2} \gamma(\omega) d \omega .
\end{aligned}
$$

Taking into account the fact that $\widehat{\Phi^{S}}=\chi_{[-\pi, \pi]}$, we derive

$$
\mathbb{E}\left[\left|X(k)-\int_{\mathbb{R}} X(t) \Phi^{S}(t-k) d t\right|^{2}\right]=0,
$$


and thus,

$$
X(k)=\int_{\mathbb{R}} X(t) \Phi^{S}(t-k) d t
$$

in quadratic mean.

$\mathbf{E}$

Lemma 1 With the same notations as in section 2.1, and for $n=\sum_{\ell=1}^{j} \epsilon_{\ell} 2^{j-\ell}$, we have

$$
\widehat{W_{n}}(\omega)=\left[\prod_{\ell=1}^{j} m_{\epsilon_{\ell}}\left(\frac{\omega}{2^{j+1-\ell}}\right)\right] \widehat{\Phi}\left(\frac{\omega}{2^{j}}\right) .
$$

\section{Proof:}

According to (5), for $\epsilon \in\{0,1\}$, we have

$$
\widehat{W_{2 n+\epsilon}}(\omega)=m_{\epsilon}\left(\frac{\omega}{2}\right) \widehat{W_{n}}\left(\frac{\omega}{2}\right) .
$$

By taking of account (28), we obtain successively

$$
\begin{aligned}
\widehat{W_{n}}(\omega) & =m_{\epsilon_{j}}\left(\frac{\omega}{2}\right) \widehat{W_{\frac{n-\epsilon_{j}}{2}}}\left(\frac{\omega}{2}\right), \\
& =m_{\epsilon_{j}}\left(\frac{\omega}{2}\right) m_{\epsilon_{j-1}}\left(\frac{\omega}{2^{2}}\right) \cdots m_{\epsilon_{1}}\left(\frac{\omega}{2^{j}}\right) \widehat{\Phi}\left(\frac{\omega}{2^{j}}\right),
\end{aligned}
$$

and thus

$$
\widehat{W}_{n}(\omega)=\left[\prod_{\ell=1}^{j} m_{\epsilon_{\ell}}\left(\frac{\omega}{2^{j+1-\ell}}\right)\right] \widehat{\Phi}\left(\frac{\omega}{2^{j}}\right) .
$$

\section{References}

[1] A. H. Tewfik and M. Kim, "Correlation structure of the discrete wavelet coefficients of fractional brownian motion," IEEE Transactions on Information Theory, vol. 38, no. 2, pp. 904-909, Mar. 1992.

[2] P. Flandrin, "Wavelet analysis and synthesis of fractional brownian motion," IEEE Transactions on Information Theory, vol. 38, no. 2, pp. 910917, Mar. 1992.

[3] A. Cohen, Ondelettes et traitement numérique du signal. Masson, Paris, 1992.

[4] R. W. Dijkerman and R. R. Mazumdar, "On the correlation structure of the wavelet coefficients of fractional brownian motion," IEEE Transactions on Information Theory, vol. 40, no. 5, pp. 1609-1612, Sep. 1994.

[5] G. G. Walter, Wavelets and other orthogonal systems with applications. CRC Press, 1994. 
[6] C. Houdré, "Wavelets, probability, and statistics: Some bridges," in Wavelets : Mathematics and applications, J. J. Benedetto and M. W. Frasier, Eds. CRC Press, 1994, ch. 9, pp. 365-398.

[7] P. F. Craigmile and D. B. Percival, "Assymptotic decorrelation of betweenscale wavelet coefficients," IEEE Transactions on Information Theory, vol. 51, no. 3, pp. 1039-1048, Mar. 2005.

[8] C. Lemoine and D. Pastor, "Démodulation cohérente par analyse multirésolution en présence de bruit parasite coloré et gaussien," Traitement $d u$ Signal, vol. 15, no. 3, pp. 251-264, 1998.

[9] J. Zhang and G. Walter, "A wavelet-based KL-like expansion for wide-sense stationary random processes," IEEE Transactions on Signal Processing, vol. 42, no. 7, pp. 1737-1745, July 1994.

[10] D. Leporini and J.-C. Pesquet, "High-order wavelet packets and cumulant field analysis," IEEE Transactions on Information Theory, vol. 45, no. 3, pp. 863-877, Apr. 1999.

[11] D. Pastor and R. Gay, "Décomposition d'un processus stationnaire du second ordre : Propriétés statistiques d'ordre 2 des coefficients d'ondelettes et localisation frequentielle des paquets d'ondelettes," Traitement du Signal, vol. 12 , no. $5,1995$.

[12] R. R. Coifman and M. V. Wickerhauser, "Entropy-based algorithms for best basis selection," IEEE Transactions on Information Theory, vol. 38, no. 2, pp. 713-718, Mar. 1992.

[13] I. Daubechies, Ten lectures on wavelets. SIAM, Philadelphie, PA, 1992.

[14] S. Mallat, A wavelet tour of signal processing, second edition. Academic Press, 1999.

[15] A. Isar and I. Naforniţă, Représentations temps-frequence. Ed Politehnica, Timişoara, Roumanie, 1998.

[16] Y. Meyer, Wavelets, Algorithms and Applications. SIAM, Philadelphie, 1993.

[17] M. V. Wickerhauser, Adapted Wavelet Analysis from Theory to Software. AK Peters, 1994.

[18] N. Saito and G. Beylkin, "Multiresolution representation using the autocorrelation functions of compactly supported wavelets," IEEE Transactions on Signal Processing, vol. 41, pp. 3584-3590, Dec. 1993.

[19] J. Shen and G. Strang, "Asymptotic analysis of Daubechies polynomials," Proceedings of the American Mathematical Society, vol. 124, no. 12, pp. 3819-3833, December 1996.

[20] — "Asymptotics of daubechies filters, scaling functions, and wavelets," Applied and Computational Harmonic Analysis, vol. 5, no. HA970234, pp. $312+, 1998$. 
[21] A. Aldroubi, M. Unser, and M. Eden, "Cardinal spline filters: Stability and convergence to the ideal sinc interpolator," Signal Process, vol. 28, no. 8, pp. 127-138, Aug. 1992.

[22] A. C. Cristán and A. T. Walden, "Multitaper power spectrum estimation and thresholding: Wavelet packets versus wavelets," IEEE Transactions on Signal Processing, vol. 50, no. 12, pp. 2976-2986, Dec. 2002.

[23] J. Yu and S. Karlsson, "Local spectral analysis using wavelet packets," Circuits Systems Signal Processing, vol. 20, no. 5, pp. 497-528, 2001.

[24] F. Gabbanini, M. Vannucci, G. Bartoli, and A. Moro, "Wavelet packet methods for the analysis of variance of time series with application to crack widths on the brunelleschi dome," Journal of Computational and Graphical Statistics, vol. 13, no. 3, pp. 639-658, 2004.

[25] D. L. Donoho and I. M. Johnstone, "Ideal adaptation by wavelet shrinkage," Biometrica, vol. 81, no. 3, pp. 425-455, Aug. 1994.

[26] D. L. Donoho, "De-noising by soft-thresholding," IEEE Transactions on Information Theory, vol. 41, no. 3, pp. 613-627, May 1995.

[27] A. Jalobeanu, L. Blanc-Féraud, and J. Zerubia, "Satellite image deconvolution using wavelet packets," INRIA, Research Report 3955, 2000.

[28] D. Daly, C. Heneghan, A. Fagan, and M. Vetterli, "Optimal wavelet packet modulation under finite complexity constraint," ICASSP, vol. 3, pp. 2789 2792, 2002.

[29] E. Kjeldsen, J. C. Dill, and A. R. Lindsey, "Exploiting the synergies of circular simplex turbo block coding and wavelet packet modulation," IEEE MILCOM, vol. 2, pp. 1202-1207, 2003.

[30] M. You and J. Ilow, "A multi-wavelet packet modulation in wireless communications," CCECE, vol. 4, pp. 2367-2370, 2004.

[31] S. Ciolino, M. Ghavani, and H. Aghvami, "On the use of wavelet packets in ultra wideband pulse shape modulation systems," IEICE, vol. E88-A, pp. 2310-2317, Sep. 2005.

[32] S. Touati and J.-C. Pesquet, "Some results on the wavelet packet decomposition of nonstationary processes," EURASIP Journal on Applied Signal Processing, vol. 2002, no. 11, pp. 1289-1295, Nov. 2002.

[33] M. Loève, Probability Theory, 4th ed. Springer-Verlag, New York, 1977.

[34] I. I. Gikhman and A. V. Skorokhod, Introduction to the theory of random processes. W. B. Saunders Company, 1969. 